\title{
SentencePiece: A simple and language independent subword tokenizer and detokenizer for Neural Text Processing
}

\author{
Taku Kudo \\ John Richardson \\ Google, Inc. \\ \{taku, johnri\}@google.com
}

\begin{abstract}
This paper describes SentencePiece, a language-independent subword tokenizer and detokenizer designed for Neural-based text processing, including Neural Machine Translation. It provides open-source $\mathrm{C}++$ and Python implementations for subword units. While existing subword segmentation tools assume that the input is pre-tokenized into word sequences, SentencePiece can train subword models directly from raw sentences, which allows us to make a purely end-to-end and language independent system. We perform a validation experiment of NMT on English-Japanese machine translation, and find that it is possible to achieve comparable accuracy to direct subword training from raw sentences. We also compare the performance of subword training and segmentation with various configurations. SentencePiece is available under the Apache 2 license at https://github.com/google/sentencepiece.
\end{abstract}

\section{Introduction}

Deep neural networks are demonstrating a large impact on Natural Language Processing. Neural machine translation (NMT) (Bahdanau et al., 2014; Luong et al., 2015; Wu et al., 2016; Vaswani et al., 2017) has especially gained increasing popularity, as it can leverage neural networks to directly perform translations with a simple end-to-end architecture. NMT has shown remarkable results in several shared tasks (Denkowski and Neubig, 2017; Nakazawa et al., 2017), and its effective approach has had a strong influence on other related NLP tasks such as dialog generation (Vinyals and Le, 2015) and automatic summarization (Rush et al., 2015).

Although NMT can potentially perform end-toend translation, many NMT systems are still relying on language-dependent pre- and postproces- sors, which have been used in traditional statistical machine translation (SMT) systems. Moses ${ }^{1}$, a de-facto standard toolkit for SMT, implements a reasonably useful pre- and postprocessor. However, it is built upon hand-crafted and language dependent rules whose effectiveness for NMT has not been proven. In addition, these tools are mainly designed for European languages where words are segmented with whitespaces. To train NMT systems for non-segmented languages such as Chinese, Korean and Japanese, we need to run word segmenters independently. Such languagedependent processing also makes it hard to train multilingual NMT models (Johnson et al., 2016), as we have to carefully manage the configurations of pre- and postprocessors per language, while the internal deep neural architectures are languageindependent.

As NMT approaches are standardized and moving forward to more language-agnostic architectures, it is becoming more important for the NLP community to develop a simple, efficient, reproducible and language independent pre- and postprocessor that can easily be integrated into Neural Network-based NLP systems, including NMT.

In this demo paper, we describe SentencePiece, a simple and language independent text tokenizer and detokenizer mainly for Neural Networkbased text generation systems where the size of vocabulary is predetermined prior to the Neural model training. SentencePiece implements two subword segmentation algorithms, byte-pairencoding (BPE) (Sennrich et al., 2016) and unigram language model (Kudo, 2018), with the extension of direct training from raw sentences. SentencePiece enables building a purely end-to-end system that does not depend on any languagespecific processing.

\footnotetext{
${ }^{1}$ http://www.statmt.org/moses/
} 


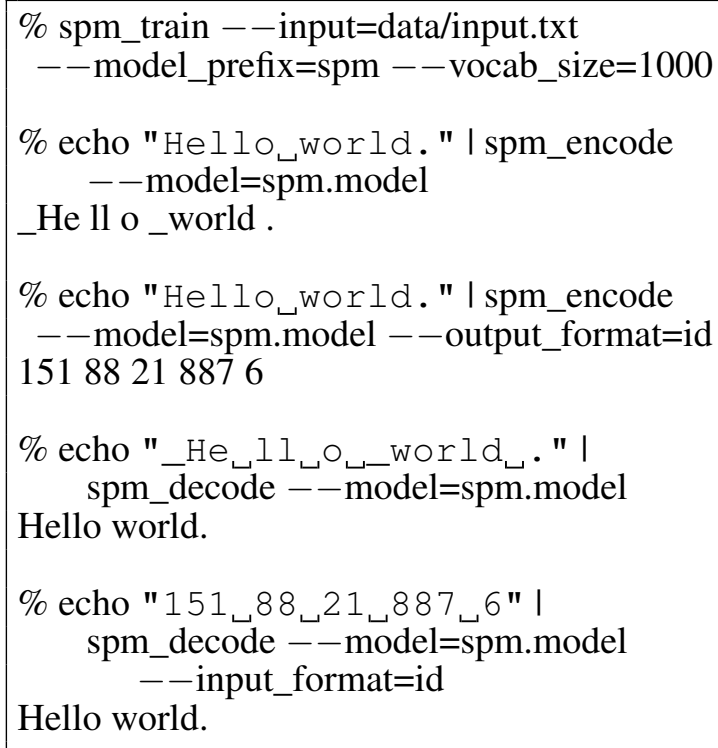

Figure 1: Commandline usage of SentencePiece

\section{System Overview}

SentencePiece comprises four main components: Normalizer, Trainer, Encoder, and Decoder. Normalizer is a module to normalize semanticallyequivalent Unicode characters into canonical forms. Trainer trains the subword segmentation model from the normalized corpus. We specify a type of subword model as the parameter of Trainer. Encoder internally executes Normalizer to normalize the input text and tokenizes it into a subword sequence with the subword model trained by Trainer. Decoder converts the subword sequence into the normalized text.

The roles of Encoder and Decoder correspond to preprocessing (tokenization) and postprocessing (detokenization) respectively. However, we call them encoding and decoding as SentencePiece manages the vocabulary to id mapping and can directly convert the text into an id sequence and vice versa. Direct encoding and decoding to/from id sequences are useful for most of NMT systems as their input and output are id sequences.

Figure 1 presents end-to-end example of SentencePiece training (spm_train), encoding (spm_encode), and decoding (spm_decode). We can see that the input text is reversibly converted through spm_encode and spm_decode.

\section{Library Design}

This section describes the design and implementation details of SentencePiece with command line and code snippets.

\subsection{Lossless Tokenization}

The following raw and tokenized sentences are an example of language-dependent preprocessing.

- Raw text: Hello world.

- Tokenized: [Hello] [world] [.]

One observation is that the raw text and tokenized sequence are not reversibly convertible. The information that no space exists between "world" and "." is not kept in the tokenized sequence. Detokenization, a process to restore the original raw input from the tokenized sequence, has to be language-dependent due to these irreversible operations. For example, while the detokenizer usually puts whitespaces between the primitive tokens in most European languages, no spaces are required in Japanese and Chinese.

- Raw text: こんにちは世界。(Hello world.)

・ Tokenized: [こんにちは] [世界] [。]

Such language specific processing has usually been implemented in manually crafted rules, which are expensive to write and maintain.

SentencePiece implements the Decoder as an inverse operation of Encoder, i.e.,

$$
\begin{array}{r}
\text { Decode }(\text { Encode }(\text { Normalize }(\text { text })))= \\
\text { Normalize }(\text { text }) .
\end{array}
$$

We call this design lossless tokenization, in which all the information to reproduce the normalized text is preserved in the encoder's output. The basic idea of lossless tokenization is to treat the input text just as a sequence of Unicode characters. Even whitespace is handled as a normal symbol. For the sake of clarity, SentencePiece first escapes the whitespace with a meta symbol _ $(U+2581)$, and tokenizes the input into an arbitrary subword sequence, for example:

- Raw text: Hello_world.

- Tokenized: [Hello] [_wor] [ld] [.]

As the whitespace is preserved in the tokenized text, we can detokenize the tokens without any ambiguities with the following Python code.

detok $=$ ' ' $\cdot$ join (tokens) .replace $\left({ }^{\prime}{ }^{\prime}{ }^{\prime}\right.$, ' ' ')

It should be noted that subword-nmt ${ }^{2}$ adopts a different representation for subword units. It focuses on how the word is segmented into subwords and uses @ @ as an intra-word boundary marker.

\footnotetext{
${ }^{2}$ https://github.com/rsennrich/subword-nmt
} 


\section{- Tokenized: [Hello] [wor] [@@ld] [@@.]}

This representation can not always perform lossless tokenization, as an ambiguity remains in the treatment of whitespaces. More specifically, it is not possible to encode consecutive whitespaces with this representation.

\subsection{Efficient subword training and segmentation}

Existing subword segmentation tools train subword models from pre-tokenized sentences. Such pre-tokenization was introduced for an efficient subword training (Sennrich et al., 2016). However, we can not always assume that pre-tokenization is available, especially for non-segmented languages. In addition, pretokenization makes it difficult to perform lossless tokenization.

SentencePiece employs several speed-up techniques both for training and segmentation to make lossless tokenization with a large amount of raw data. For example, given an input sentence (or word) of length $N$, BPE segmentation requires $O\left(N^{2}\right)$ computational cost when we naively scan the pair of symbols in every iteration. SentencePiece adopts an $O(N \log (N))$ algorithm in which the merged symbols are managed by a binary heap (priority queue). In addition, the training and segmentation complexities of unigram language models are linear to the size of input data.

\subsection{Vocabulary id management}

SentencePiece manages the vocabulary to id mapping to directly convert the input text into an id sequence and vice versa. The size of vocabulary is specified with the--vocab_size=<size $>$ flag of spm_train. While subword-nmt specifies the number of merge operations, SentencePiece specifies the final size of vocabulary, as the number of merge operations is a BPE specific parameter and can not be applicable to other segmentation algorithms, e.g., unigram language model (Kudo, 2018).

SentencePiece reserves vocabulary ids for special meta symbols, e.g., unknown symbol $(<$ unk $>)$, BOS $(<s>)$, EOS $(</ s>)$ and padding $(<$ pad $>)$. Their actual ids are configured with command line flags. We can also define custom meta symbols to encode contextual information as virtual tokens. Examples include the languageindicators, $<2 j a>$ and $<2 d e>$, for multilingual
$\mathrm{U}+41 \mathrm{U}+302 \mathrm{U}+300<\mathrm{tab}>\mathrm{U}+1 \mathrm{EA} 6$

$\mathrm{U}+41 \mathrm{U}+302 \mathrm{U}+301<\mathrm{tab}>\mathrm{U}+1 \mathrm{EA} 4$

$\cdots$

Figure 2: Custom normalization rule in TSV

models (Johnson et al., 2016).

\subsection{Customizable character normalization}

Character normalization is an important preprocessing step for handling real world text, which consists of semantically-equivalent Unicode characters. For example, Japanese fullwidth Latin characters can be normalized into ASCII Latin characters. Lowercasing is also an effective normalization, depending on the application.

Character normalization has usually been implemented as hand-crafted rules. Recently, Unicode standard Normalization Forms, e.g., NFC and NFKC, have been widely used in many NLP applications because of their better reproducibility and strong support as Unicode standard.

By default, SentencePiece normalizes the input text with the Unicode NFKC normalization. The normalization rules are specified with the --normalization_rule_name=nfkc flag of spm_train. The normalization in Sentencepiece is implemented with string-to-string mapping and leftmost longest matching. The normalization rules are compiled into a finite state transducer (Aho-Corasick automaton) to perform an efficient normalization ${ }^{3}$.

SentencePiece supports custom normalization rules defined as a TSV file. Figure 2 shows an example TSV file. In this example, the Unicode sequence [U+41 $U+302 \quad \mathrm{U}+300]$ is converted into $\mathrm{U}+1 \mathrm{EA} 6^{4}$. When there are ambiguities in the conversion, the longest rule is applied. User defined TSV files are specified with the --normalization_rule_tsv=<file $>$ flag of spm_train. Task-specific rules can be defined by extending the default NFKC rules provided as a TSV file in SentencePiece package.

\subsection{Self-contained models}

Recently, many researchers have provided pretrained NMT models for better reproduciblity of

\footnotetext{
${ }^{3}$ The original NFKC normalization requires CCC (Canonical Combining Class) reordering, which is hard to model in a finite state transducer. SentencePiece does not handle the full CCC reordering and only implements a subset of NFKC normalization.

${ }^{4}$ Note that tabs are used as the delimiter for source and target sequence and spaces are used as the delimiter for individual characters.
} 
their experimental results. However, it is not always stated how the data was preprocessed. (Post, 2018) reported that subtle differences in preprocessing schemes can widely change BLEU scores. Even using the Moses toolkit, it is not guaranteed to reproduce the same settings unless the configurations of Moses (e.g., version and command line flags) are clearly specified. Strictly speaking, NFKC normalization may yield different results depending on the Unicode version.

Ideally, all the rules and parameters for preprocessing must be embedded into the model file in a self-contained manner so that we can reproduce the same experimental setting as long as we are using the same model file.

The SentencePiece model is designed to be purely self-contained. The model file includes not only the vocabulary and segmentation parameters, but also the pre-compiled finite state transducer for character normalization. The behavior of SentencePiece is determined only by the model file and has no external dependencies. This design guarantees a perfect reproducibility as well as allowing to distribute the SentencePiece model file as part of an NMT model. In addition, the developers of SentencePiece can refine the (default) normalization rules without having to worry about breaking existing preprocessing behaviors.

The SentencePiece model is stored as a binary wire format Protocol buffer ${ }^{5}$, a platform neutral and extensible mechanism for serializing structured data. Protocol buffers help to safely serialize structured data while keeping backward compatibility as well as extensibility.

\subsection{Library API for on-the-fly processing}

Text preprocessing is usually considered as offline processing. Prior to the main NMT training, raw input is preprocessed and converted into an id sequence with a standalone preprocessor.

Such off-line preprocessing has two problems. First, standalone tools are not directly integrated into the user-facing NMT applications which need to preprocess user input on-the-fly. Second, offline preprocessing makes it hard to employ subsentence level data augmentation and noise injection, which aim at improving the accuracy and robustness of the NMT models. There are several studies to inject noise to input sen-

\footnotetext{
${ }^{5}$ https: / / developers.google.com/ protocol-buffers/
}

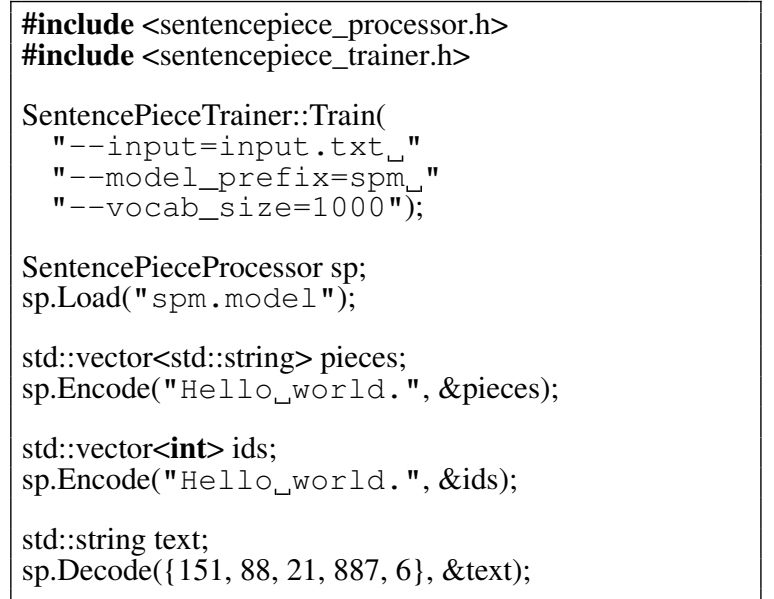

Figure 3: C++ API usage (The same as Figure 1.)

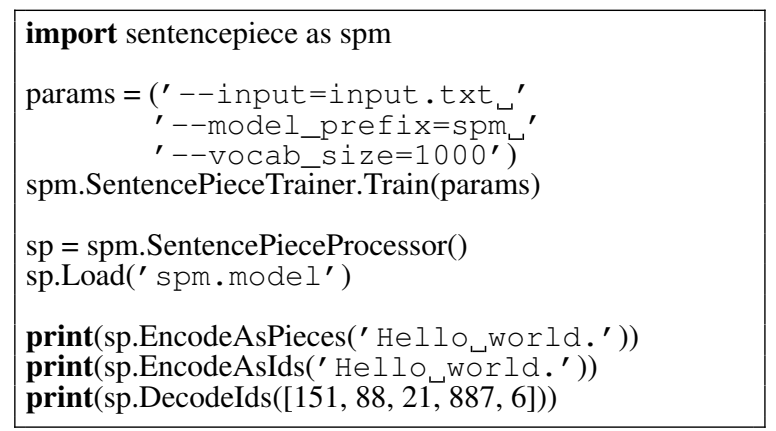

Figure 4: Python API usage (The same as Figure 1.)

tences by randomly changing the internal representation of sentences. (Kudo, 2018) proposes a subword regularization that randomly changes the subword segmentation during NMT training. (Lample et al., 2017; Artetxe et al., 2017) independently proposed a denoising autoencoder in the context of sequence-to-sequence learning, where they randomly alter the word order of the input sentence and the model is trained to reconstruct the original sentence. It is hard to emulate this dynamic sampling and noise injection only with the off-line processing.

SentencePiece not only provides a standalone command line tool for off-line preprocessing but supports a C++, Python and Tensorflow library API for on-the-fly processing, which can easily be integrated into existing NMT frameworks. Figures 3, 4 and 5 show example usages of the $\mathrm{C}++$, Python and TensorFlow API ${ }^{6}$. Figure 6 presents example Python code for subword regularization where one subword sequence is sampled according to the unigram language model. We can find that the text "New York" is tokenized differently

\footnotetext{
${ }^{6}$ As the Python and TensorFlow wrappers call the native $\mathrm{C}++\mathrm{API}$, there is no performance drop in their interfaces.
} 


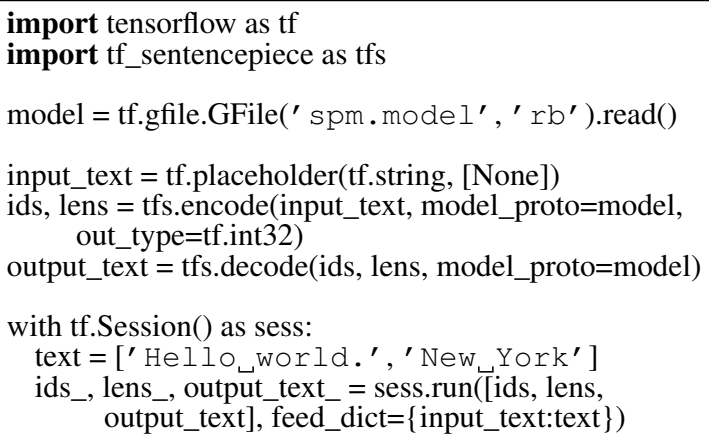

Figure 5: TensorFlow API usage

The SentencePiece model (model proto) is an attribute of the TensorFlow operation and embedded into the TensorFlow graph so the model and graph become purely self-contained.

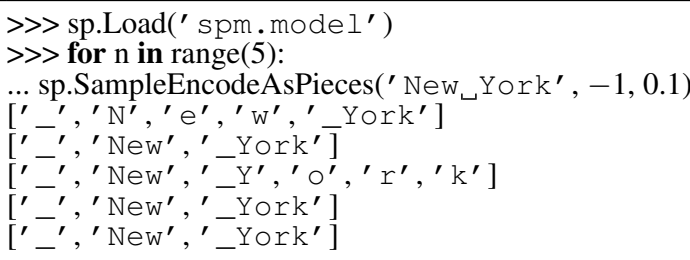

Figure 6: Subword sampling with Python API

on each SampleEncodeAsPieces call. Please see (Kudo, 2018) for the details on subword regularization and its sampling hyperparameters.

\section{Experiments}

\subsection{Comparison of different preprocessing}

We validated the performance of the different preprocessing on English-Japanese translation of Wikipedia articles, as specified by the Kyoto Free Translation Task (KFTT) ${ }^{7}$. The training, development and test data of KFTT consist of 440k, 1166 and 1160 sentences respectively.

We used GNMT (Wu et al., 2016) as the implementation of the NMT system in our experiments. We generally followed the settings and training procedure described in (Wu et al., 2016), however, we changed the node and layer size of LSTM to be 512 and 6 respectively.

A word model is used as a baseline system. We compared to SentencePiece (unigram language model) with and without pre-tokenization. SentencePiece with pre-tokenization is essentially the same as the common NMT configuration with subword-nmt. SentencePiece without pretokenization directly trains the subword model from raw sentences and does not use any external resources. We used the Moses tokenizer ${ }^{8}$ and

\footnotetext{
${ }^{7}$ http: //Www. phontron. com/kftt

${ }^{8}$ http: //www. statmt.org/moses/
}

\begin{tabular}{l|l|l|l}
\hline Lang pair & setting (source/target) & \# vocab. & BLEU \\
\hline ja $\rightarrow$ en & Word model (baseline) & $80 \mathrm{k} / 80 \mathrm{k}$ & 28.24 \\
& SentencePiece & $8 \mathrm{k}$ (shared) & 29.55 \\
& SentencePiece w/ pre-tok. & $8 \mathrm{k}$ (shared) & 29.85 \\
& Word/SentencePiece & $80 \mathrm{k} / 8 \mathrm{k}$ & 27.24 \\
& SentencePiece/Word & $8 \mathrm{k} / 80 \mathrm{k}$ & 29.14 \\
\hline en $\rightarrow$ ja & Word model (baseline) & $80 \mathrm{k} / 80 \mathrm{k}$ & 20.06 \\
& SentencePiece & $8 \mathrm{k}$ (shared) & 21.62 \\
& SentencePiece w/ pre-tok. & $8 \mathrm{k}$ (shared) & 20.86 \\
& Word/SentencePiece & $80 \mathrm{k} / 8 \mathrm{k}$ & 21.41 \\
& SentencePiece/Word & $8 \mathrm{k} / 80 \mathrm{k}$ & 19.94 \\
\hline
\end{tabular}

Table 1: Translation Results (BLEU(\%))

KyTea ${ }^{9}$ for English and Japanese pre-tokenization respectively. The same tokenizers are applied to the word model.

We used the case-sensitive BLEU score (Papineni et al., 2002) as an evaluation metric. As the output sentences are not segmented in Japanese, we segmented them with KyTea for before calculating BLEU scores.

Table 1 shows the experimental results. First, as can be seen in the table, subword segmentations with SentencePiece consitently improve the BLEU scores compared to the word model. This result is consistent with previous work (Sennrich et al., 2016). Second, it can be seen that the pre-tokenization is not always necessary to boost the BLEU scores. In Japanese to English, the improvement is marginal and has no significant difference. In English to Japanese, the BLEU score is degraded with pre-tokenization.

We can find larger improvements in BLEU when 1) SentencePiece is applied to Japanese, and 2) the target sentence is Japanese. As Japanese is a non-segmented language, pre-tokenization acts as a strong constraint to determine the final vocabulary. It can be considered that the positive effects of unsupervised segmentation from raw input worked effectively to find the domain-specific vocabulary in Japanese.

\subsection{Segmentation performance}

Table 2 summarizes the training and segmentation performance of various configurations.

We can see that the training and segmentation speed of both SentencePiece and subword-nmt is almost comparable on English data set regardless of the choice of pre-tokenization. This is expected, as English is a segmented language and the search space for the vocabulary extraction is largely restricted. On the other hand, SentencePiece shows

\footnotetext{
${ }^{9}$ http://www. phontron. com/kytea
} 


\begin{tabular}{l|c|c|r|r}
\hline \multirow{2}{*}{ Task } & & & \multicolumn{2}{|c}{ time (sec.) } \\
\cline { 4 - 5 } Train & Tool & Pre-tok. & Japanese & English \\
& subword-nmt & yes & 56.9 & 54.1 \\
& SentencePiece & yes & 10.1 & 16.8 \\
& subword-nmt & no & 528.0 & 94.7 \\
& SentencePiece & no & 217.3 & 21.8 \\
\hline \multirow{2}{*}{ Seg. } & subword-nmt & yes & 23.7 & 28.6 \\
& SentencePiece & yes & 8.2 & 20.3 \\
& subword-nmt & no & 216.2 & 36.1 \\
& SentencePiece & no & 5.9 & 20.3 \\
\hline \multicolumn{2}{l}{ Pre-tokenizaion KyTea(ja)/Moses(en) } & 24.6 & 15.8 \\
\hline
\end{tabular}

Table 2: Segmentation performance. KFTT corpus $(440 \mathrm{k}$ sentences) is used for evaluation. Experiments are executed on Linux with Xeon 3.5Ghz processors. The size of vocabulary is 16k. Moses and KyTea tokenizers are used for English and Japanese respectively. Note that we have to take the time of pre-tokenization into account to make a fair comparison with and without pre-tokenization. Because subword-nmt is based on BPE, we used the BPE model in SentencePiece. We found that BPE and unigram language models show almost comparable performance.

larger performance improvements when applying it to raw Japanese data (w/o pre-tok). The segmentation speed of SentencePiece is about 380 times faster than that of subword-nmt in this setting. This result strongly supports our claim that SentencePiece is fast enough to be applied to raw data and the pre-tokenization is not always necessary. Consequently, SentencePiece helps to build a purely data-driven and language-independent system. The segmentation speed of SentencePiece is around 21k and 74k sentences/sec. in English and Japanese respectively, which is fast enough to be executed on-the-fly.

\section{Conclusions}

In this paper, we introduced SentencePiece, an open-source subword tokenizer and detokenizer designed for Neural-based text processing. SentencePiece not only performs subword tokenization, but directly converts the text into an id sequence, which helps to develop a purely end-toend system without replying on language specific resources. The model file of SentencePiece is designed to be self-contained to guarantee perfect reproducibility of the normalization and subword segmentation. We hope that SentencePiece will provide a stable and reproducible text processing tool for production use and help the research community to move to more language-agnostic and multilingual architectures.

\section{References}

Mikel Artetxe, Gorka Labaka, Eneko Agirre, and Kyunghyun Cho. 2017. Unsupervised neural machine translation. arXive preprint arXiv:1710.11041.

Dzmitry Bahdanau, Kyunghyun Cho, and Yoshua Bengio. 2014. Neural machine translation by jointly learning to align and translate. arXiv preprint arXiv:1409.0473.

Michael Denkowski and Graham Neubig. 2017. Stronger baselines for trustable results in neural machine translation. Proc. of Workshop on Neural Machine Translation.

Melvin Johnson, Mike Schuster, et al. 2016. Google's multilingual neural machine translation system: enabling zero-shot translation. arXiv preprint arXiv:1611.04558.

Taku Kudo. 2018. Subword regularization: Improving neural network translation models with multiple subword candidates. In Proc. of ACL.

Guillaume Lample, Ludovic Denoyer, and Marc'Aurelio Ranzato. $2017 . \quad$ Unsupervised machine translation using monolingual corpora only. arXive preprint arXiv:1711.00043.

Minh-Thang Luong, Hieu Pham, and Christopher D Manning. 2015. Effective approaches to attentionbased neural machine translation. In Proc of EMNLP.

Toshiaki Nakazawa, Shohei Higashiyama, et al. 2017. Overview of the 4th workshop on asian translation. In Proceedings of the 4th Workshop on Asian Translation (WAT2017).

Kishore Papineni, Salim Roukos, Todd Ward, and WeiJing Zhu. 2002. Bleu: a method for automatic evaluation of machine translation. In Proc. of ACL.

Matt Post. 2018. A call for clarity in reporting bleu scores. arXiv preprint arXiv:1804.08771.

Alexander M Rush, Sumit Chopra, and Jason Weston. 2015. A neural attention model for abstractive sentence summarization. In Proc. of EMNLP.

Rico Sennrich, Barry Haddow, and Alexandra Birch. 2016. Neural machine translation of rare words with subword units. In Proc. of ACL.

Ashish Vaswani, Noam Shazeer, Niki Parmar, Jakob Uszkoreit, Llion Jones, Aidan N. Gomez, Lukasz Kaiser, and Illia Polosukhin. 2017. Attention is all you need. arXive preprint arXiv:1706.03762.

Oriol Vinyals and Quoc V. Le. 2015. A neural conversational model. In ICML Deep Learning Workshop.

Yonghui Wu, Mike Schuster, et al. 2016. Google's neural machine translation system: Bridging the gap between human and machine translation. arXiv preprint arXiv:1609.08144. 